\title{
Uncertainty and reliability in aircraft design and optimization
}

\author{
S. Hernández, J. Díaz, M. Cid, A. Baldomir \& L. Romera \\ Structural Mechanics Group, School of Civil Engineering, \\ University of La Coruña, Spain
}

\begin{abstract}
Aircraft engineering is subjected to many classes of uncertainties due to the lack of proper definition of loads, behaviour of new materials or even due to the inaccuracies produced during manufacturing. Because of that, the most advanced methods of analysis and optimization need to be used during the dimensioning of aircraft structures. One way to increase the safety level of a design could be to increase the safety coefficients for load values or material strength, but this approach would lead to an unacceptable amount of material for the aircraft. More proper approaches can be applied using probabilistic analysis during the design phase. In that case, some of the parameters, such as loads, material properties of manufacturing tolerances are defined as random variables and a probabilistic analysis is carried out to identify the safety of the design. This approach can be also enhanced by introducing the concept of design optimization. In that case the optimum solution for an aircraft structure is obtained even considering the random nature of some of the design variables. In this paper these methodologies will be described and some examples of aircraft structures will be presented to show the potential in real problems.

Keywords: uncertainty quantification, reliability based design optimization.
\end{abstract}

\section{Introduction}

Reliability is related with the probability of verifying a certain condition. This is known as the probability of failure. In a probabilistic analysis, the uncertainties in the basic magnitudes of the structure are considered directly in the analysis, changing from fixed quantities to random variables (RV). The limit state function defines if a design belongs to the failure domain, where the limit state is not 
verified, or to the security domain, where it is (figure 1). If $\mathbf{a}$ is the vector of basic variables which contains the $n$ random variables of the structure, then the domains are defined as follows:

$$
\begin{gathered}
\text { Failure domain: } F=\{\mathbf{a} \mid g(\mathbf{a})<0\} \\
\text { Security domain: } S=\{\mathbf{a} \mid g(\mathbf{a}) \geqslant 0\}
\end{gathered}
$$

The boundary between both domains is known as the failure surface or limit state surface, which generally is an hypersurface of $n-1$ dimensions in the $n$ dimensional space of basic variables. The safety margin is now defined as a random variable which can be identified with the value of the limit state function:

$$
M=g(\mathbf{a})
$$

According to this, probability of failure $p_{f}$ is formulated as:

$$
p_{f}=P[g(\mathbf{a}) \leqslant 0]=\int \underset{g(\mathbf{a}) \leqslant 0}{\ldots} f_{\mathbf{A}}(\mathbf{a}) d \mathbf{a},
$$

where $f_{\mathbf{A}}(\mathbf{a})$ is the joint probability density function of all the basic variables involved in the response of the system. Except in some particular cases, integral expression (4) cannot be resolved analytically, because of the nonlinearity of $f_{\mathbf{A}}(\mathbf{a})$, and also due to the fact that the number of random variables usually employed is large, and therefore the dimension of the problem.

The uncertainty quantification methods selected in this work to assess the reliability of the structure are based on the Taylor series expansion of the limit state surface. Those methods require information about the value of the limit state function and its derivatives in the vicinity of the design point. A brief description of the algorithms is presented next.

\section{Reliability analysis methods}

\subsection{First order second-moment methods}

The FOSM or first order second-moment method uses an estimation of the first two statistical moments of the limit state function evaluated at $\boldsymbol{\mu}_{\mathrm{A}}$, the point defined by the mean value of the random variables [1]:

$$
\begin{aligned}
\mu_{M} & =E[g(\mathbf{a})] \simeq g\left(\boldsymbol{\mu}_{\mathbf{A}}\right) \\
\sigma_{M}^{2}=\operatorname{Var}[g(\mathbf{a})] & \simeq \sum_{i=1}^{n} \sum_{j=1}^{n} \frac{\partial g\left(\boldsymbol{\mu}_{\mathbf{A}}\right)}{\partial a_{i}} \frac{\partial g\left(\boldsymbol{\mu}_{\mathbf{A}}\right)}{\partial a_{j}} \sigma_{A_{i} A_{j}},
\end{aligned}
$$

where $\mu_{M}$ and $\sigma_{M}$ are the mean value and the standard deviation of the limit state function, respectively. Reliability index $\beta$ is defined from equations (5) and (6) as

$$
\beta=\frac{\mu_{M}}{\sigma_{M}}
$$




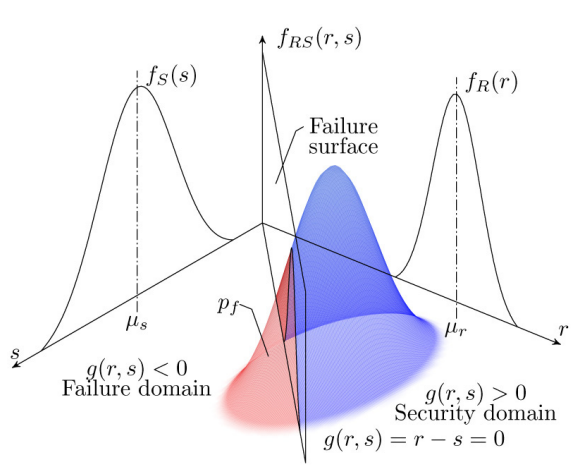

Figure 1: Failure and security domains.

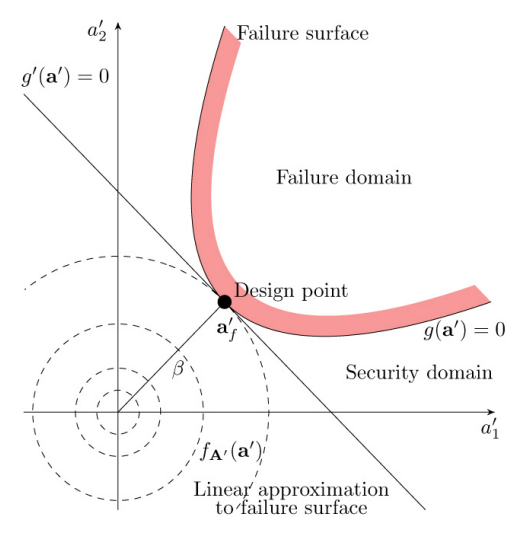

Figure 2: First order reliability method.

whereas the probability of failure $p_{f}$ can be obtained as

$$
p_{f}=1-\Phi(\beta),
$$

where $\Phi$ is the cumulative distribution function of the standard normal variable.

\subsection{First order reliability methods}

The FORM or first order reliability method [2] consists in the search of the most probable point of failure (MPP) in the standardised domain of the random variables $\left(\mathbf{a}^{\prime}\right)$, in order to allow the substitution of the limit state function by its Taylor series expansion of first order at that point (figure 2),

$$
g\left(\mathbf{a}^{\prime}\right) \simeq g\left(\mathbf{a}_{\mathbf{f}}^{\prime}\right)+\nabla g\left(\mathbf{a}_{f}^{\prime}\right)^{T}\left(\mathbf{a}^{\prime}-\mathbf{a}_{f}^{\prime}\right)
$$

where $\mathbf{a}_{f}^{\prime}$, the most probable point of failure, is the point of minimum distance from the origin to the limit state surface. Geometrically, the method approximates the limit state surface by the tangent hyperplane at the MPP. Reliability index is related now to the failure surface, but it is invariant with respect to the formulation of the limit state function:

$$
\beta=-\frac{\mathbf{a}_{f}^{\prime T} \nabla g\left(\mathbf{a}_{f}^{\prime}\right)}{\sqrt{\nabla g\left(\mathbf{a}_{f}^{\prime}\right)^{T} \nabla g\left(\mathbf{a}_{f}^{\prime}\right)}} .
$$




\subsection{Advanced mean value methods}

These algorithms assume the approximation of the limit state surface by the tangent hyperplane at the point $\boldsymbol{\mu}_{\mathbf{A}}$. The first order expansion is known as AMV:

$$
M=g(\mathbf{a}) \simeq g\left(\boldsymbol{\mu}_{\mathbf{A}}\right)+\nabla g\left(\boldsymbol{\mu}_{\mathbf{A}}\right)^{T}\left(\mathbf{a}-\boldsymbol{\mu}_{\mathbf{A}}\right),
$$

whereas $\mathrm{AMV}+$ is an enhanced first order approximation which starts in $\boldsymbol{\mu}_{\mathrm{A}}$ and then iterates to obtain an estimation of the MPP $\left(\mathbf{a}^{*}\right)$ :

$$
M=g(\mathbf{a}) \simeq g\left(\boldsymbol{a}^{*}\right)+\nabla g\left(\boldsymbol{a}^{*}\right)^{T}\left(\mathbf{a}-\boldsymbol{a}^{*}\right) .
$$

\subsection{Two-point adaptive nonlinear approximation}

The two-point adaptive nonlinear approximation (TANA3) is based on an exponential approximation which uses information of the current iteration $k$ and also of the previous one $k-1$ [3]. According to that, the limit state surface can be approximated as

$g(\mathbf{a}) \simeq g\left(\mathbf{a}_{k}\right)+\sum_{i=1}^{n} \frac{\partial g\left(\mathbf{a}_{k}\right)}{\partial a_{i}} \frac{\left(a_{i, k}\right)^{\left(1-r_{i}\right)}}{r_{i}}\left(a_{i}^{r_{i}}-a_{i, k}^{r_{i}}\right)+\frac{\varepsilon_{2}}{2} \sum_{i=1}^{n}\left(a_{i}^{r_{i}}-a_{i, k}^{r_{i}}\right)^{2}$,

where the nonlinear index $r_{i}$ and the parameter $\varepsilon_{2}$ are defined as

$$
\begin{aligned}
r_{i} & =1+\frac{\ln \left(\frac{\partial g\left(\mathbf{a}_{k-1}\right)}{\partial a_{i}}\right)-\ln \left(\frac{\partial g\left(\mathbf{a}_{k}\right)}{\partial a_{i}}\right)}{\ln \left(a_{i, k-1}\right)-\ln \left(a_{i, k}\right)}, \\
\varepsilon_{2}= & \frac{2\left[g\left(\mathbf{a}_{k-1}\right)-g\left(\mathbf{a}_{k}\right)\right]}{\sum_{i=1}^{n}\left(a_{i}^{r_{i}}-a_{i, k-1}^{r_{i}}\right)^{2}+\sum_{i=1}^{n}\left(a_{i}^{r_{i}}-a_{i, k}^{r_{i}}\right)^{2}}- \\
- & \frac{2\left[\sum_{i=1}^{n} \frac{a_{i, k}^{1-r_{i}}}{r_{i}} \frac{\partial g\left(\mathbf{a}_{k}\right)}{\partial a_{i}}\left(a_{i, k-1}^{r_{i}}-a_{i, k}^{r_{i}}\right)\right]}{\sum_{i=1}^{n}\left(a_{i}^{r_{i}}-a_{i, k-1}^{r_{i}}\right)^{2}+\sum_{i=1}^{n}\left(a_{i}^{r_{i}}-a_{i, k}^{r_{i}}\right)^{2}}
\end{aligned}
$$

\section{Reliability based design optimization}

Reliability based design optimization solves the problem of minimizing an objective function, usually related with the cost of the structure, like the weight, considering constraints defined in terms of uncertainty. The variables that characterize the problem can be divided into two types. Design variables, which are modified in the optimization cycle and random variables, which are the source of uncertainty. 
RBDO is an active research topic and many authors have contributed so far. Some interesting references can be found in [4-8].

The formulation of a RBDO problem can be set out in different ways, depending on where the procedure to evaluate the reliability constraints is connected to the optimization process. Thus, bi-level or double loop approaches consider the reliability constraints within the optimization loop. On the other hand, monolevel approaches replace probabilistic constraints with approximate deterministic values, converting the double loop in a single loop. Finally, decoupled approach solves the RBDO problem as a sequence of deterministic optimization procedures.

In this work a double loop framework with a reliability index approach has been selected as methodology. The algorithm selected for the optimization loop is sequential quadratic programming

\section{Application examples}

This section demonstrates the performance of the selected reliability methods when applied in the context of a design optimization algorithm. Three different examples are presented. In all the cases, the probabilistic results are compared with the deterministic results, using in this case the mean values of the random variables.

\subsection{Ten bar truss}

Figure 3 shows the geometry of a ten bar truss structure. Two loads of the same value are applied and modelled with a random variable using a Gumbel distribution. The mean value is $255.5 \mathrm{kN}$, and the coefficient of variation (COV) is 0.15 . The material is aluminum, having deterministic values the density and the Poisson's coefficient, with $2.77 \mathrm{t} / \mathrm{m}^{3}$ and 0.3 respectively. The Young's modulus is considered as a random variable with a normal distribution, with mean $6.895 \times 10^{4}$ $\mathrm{MPa}$ and COV 0.05. Dimensions are deterministic and the parameter $L$ is 9.144 $\mathrm{m}$. The cross section areas are the design variables. They have an initial value of $153.938 \mathrm{~cm}^{2}$, and are constrained by a lower and upper bound, with values of $10.425 \mathrm{~cm}^{2}$ and $216.111 \mathrm{~cm}^{2}$, respectively.

This structure will be optimized by means of minimizing its volume, considering deterministic optimization and reliability based design optimization. The maximum displacement in the node 5 is selected as constraint, so the deterministic problem is formulated as:

$$
\min F(\boldsymbol{x})
$$

subject to:

$$
w_{5} \leqslant w_{\max }
$$

In this case, the maximum displacement allowed on node 5 is $w_{\max }=-5.08$ $\mathrm{cm}$. When considering reliability based design optimization, the constraints are 
random quantities and the problem is formulated as:

$$
\min F(\boldsymbol{x})
$$

subject to:

$$
\beta_{i} \geqslant \beta_{\text {min }}
$$

where $\beta_{\min }=3$, which corresponds with a probability of failure of $1.35 \times 10^{-3}$.

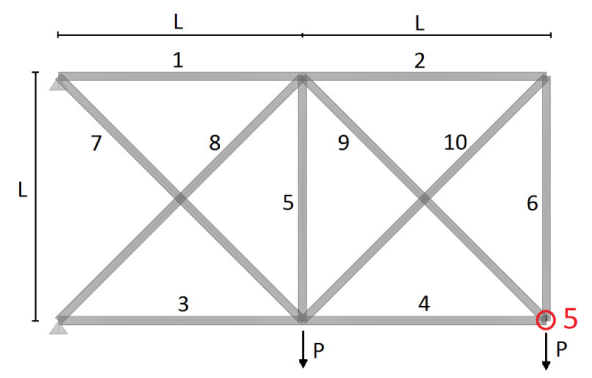

Figure 3: Ten bar truss structure.

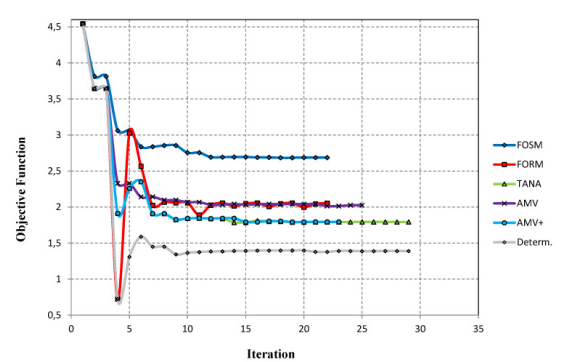

Figure 4: Ten bar truss objective function.

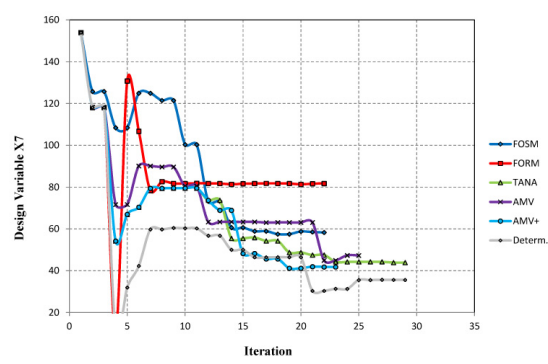

Figure 6: Convergence of design variable $x_{7}$.

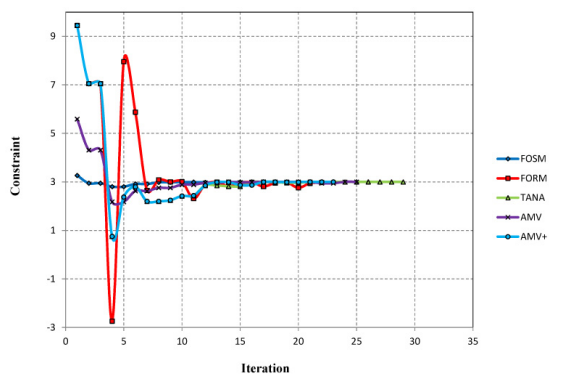

Figure 5: Ten bar truss reliability constraint.

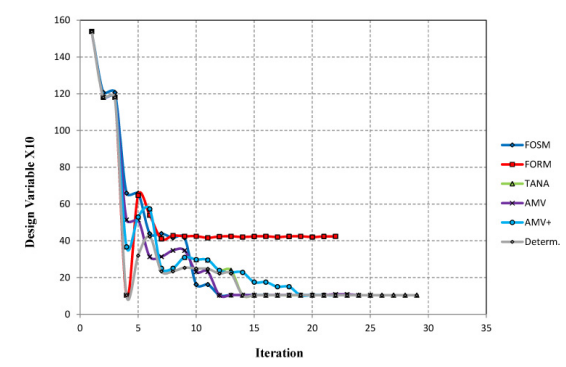

Figure 7: Convergence of design variable $x_{10}$. 
Table 1: Ten bar truss results summary.

\begin{tabular}{lcccccc}
\hline Method & FOSM & FORM & TANA & AMV & AMV+ & Determ. \\
\hline$x_{1}$ & 216.111 & 144.240 & 144.850 & 162.213 & 145.584 & 109.340 \\
$x_{2}$ & 10.452 & 43.625 & 10.452 & 10.452 & 10.452 & 10.452 \\
$x_{3}$ & 166.594 & 140.847 & 109.968 & 130.792 & 107.569 & 85.573 \\
$x_{4}$ & 109.601 & 46.627 & 67.993 & 76.687 & 68.750 & 50.003 \\
$x_{5}$ & 10.452 & 32.147 & 10.452 & 10.452 & 10.452 & 10.452 \\
$x_{6}$ & 10.452 & 43.604 & 10.452 & 10.452 & 10.452 & 10.452 \\
$x_{7}$ & 58.327 & 81.734 & 43.921 & 47.295 & 41.798 & 35.642 \\
$x_{8}$ & 159.494 & 79.664 & 99.972 & 114.812 & 102.393 & 75.551 \\
$x_{9}$ & 151.582 & 50.421 & 96.073 & 109.461 & 96.462 & 70.843 \\
$x_{10}$ & 10.452 & 42.456 & 10.452 & 10.452 & 10.452 & 10.452 \\
$F$ & 2.687 & 2.053 & 1.794 & 2.026 & 1.794 & 1.389 \\
Iterations & 22 & 22 & 29 & 25 & 23 & 29 \\
Evaluations & 3410 & 504,406 & 175,605 & 4590 & 197,676 & 869 \\
\hline
\end{tabular}

As it is shown in figures 4 to 7 and in table 1, TANA and AMV+ methods reach similar objective function values and AMV has an increase of $13 \%$ in its objective function value with respect to them. In the case of the FOSM method, the result obtained has an increase of $50 \%$ with respect to TANA and AMV+, due to the differences on the value obtained for the reliability index at the initial design. As expected, deterministic design converges into a lower value. The most expensive methods are AMV+ and TANA with 197,000 and 175,000 evaluations, respectively. The methods requiring less iterations are FOSM and AMV, with 3400 and 4600 evaluations, respectively.

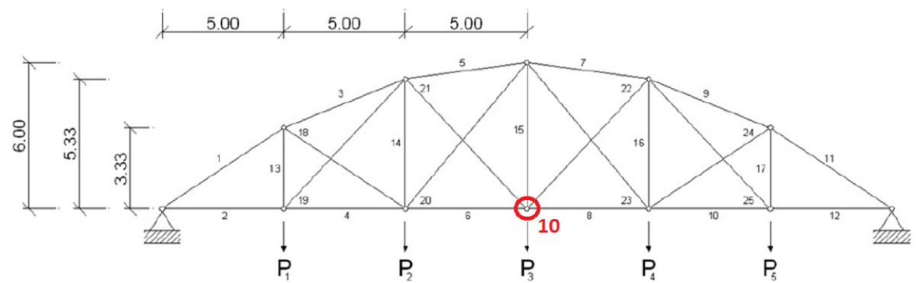

Figure 8: Twenty-five bar 2D truss structure. 
Table 2: Twenty-five bar 2D truss design variables.

\begin{tabular}{lccccccccccccc}
\hline Variable & $x_{1}$ & $x_{2}$ & $x_{3}$ & $x_{4}$ & $x_{5}$ & $x_{6}$ & $x_{7}$ & $x_{8}$ & $x_{9}$ & $x_{10}$ & $x_{11}$ & $x_{12}$ & $x_{13}$ \\
\hline Initial point & 15 & 0.5 & 15 & 0.5 & 15 & 0.5 & 2 & 0.1 & 2 & 2 & 0.1 & 0.1 & 2 \\
\hline
\end{tabular}

Table 3: Twenty-five bar 2D truss results summary.

\begin{tabular}{lcccccc}
\hline Method & FOSM & FORM & TANA & AMV & AMV+ & Determ. \\
\hline$x_{1}$ & 13.350 & 12.949 & 12.741 & 12.564 & 13.418 & 12.998 \\
$x_{2}$ & 0.100 & 0.100 & 0.100 & 0.100 & 0.100 & 0.100 \\
$x_{3}$ & 13.535 & 13.351 & 13.226 & 13.207 & 13.377 & 12.957 \\
$x_{4}$ & 0.434 & 0.439 & 0.436 & 0.432 & 0.314 & 0.315 \\
$x_{5}$ & 15.367 & 15.109 & 15.346 & 15.850 & 14.791 & 14.373 \\
$x_{6}$ & 0.100 & 0.100 & 0.100 & 0.100 & 0.100 & 0.100 \\
$x_{7}$ & 1.828 & 1.697 & 1.837 & 1.827 & 1.234 & 1.319 \\
$x_{8}$ & 0.100 & 0.100 & 0.100 & 0.100 & 0.100 & 0.100 \\
$x_{9}$ & 2.381 & 2.336 & 2.406 & 2.461 & 2.850 & 2.691 \\
$x_{10}$ & 1.741 & 1.790 & 1.761 & 1.748 & 1.172 & 1.151 \\
$x_{11}$ & 0.100 & 0.100 & 0.100 & 0.100 & 0.100 & 0.100 \\
$x_{12}$ & 0.100 & 0.100 & 0.100 & 0.100 & 0.100 & 0.100 \\
$x_{13}$ & 2.305 & 2.279 & 2.339 & 2.397 & 2.319 & 2.256 \\
$F$ & 0.433 & 0.425 & 0.426 & 0.429 & 0.421 & 0.409 \\
Iterations & 17 & 18 & 17 & 14 & 42 & 30 \\
Evaluations & 1050 & 182,109 & 58,458 & 1175 & 213,441 & 602 \\
\hline
\end{tabular}

\subsection{Twenty-five bar 2D truss}

Figure 8 shows the geometry of a 25 bar 2D truss structure. The load case in study is composed by five loads applied on the nodes of the lower bars. The value of these five loads is supposed to be equal, being a random variable with a Gumbel distribution. The mean value is $286.5 \mathrm{kN}$, and the COV is 0.1 . The material used in this truss is steel, having deterministic values the density and the Poisson's coefficient, with $7.85 \mathrm{t} / \mathrm{m}^{3}$ and 0.3 , respectively. Young's modulus is a random variable with a normal distribution, with mean $2.1 \times 10^{5} \mathrm{MPa}$ and COV 0.05. The dimensions of the truss are deterministic values and the design variables are the cross section areas of the bars, with the initial values shown in table 2, constrained by a lower and upper bound, with values $0.1 \mathrm{~cm}^{2}$ and $1000 \mathrm{~cm}^{2}$, respectively. The maximum displacement allowed on node 10 is $-2.5 \mathrm{~cm}$. 


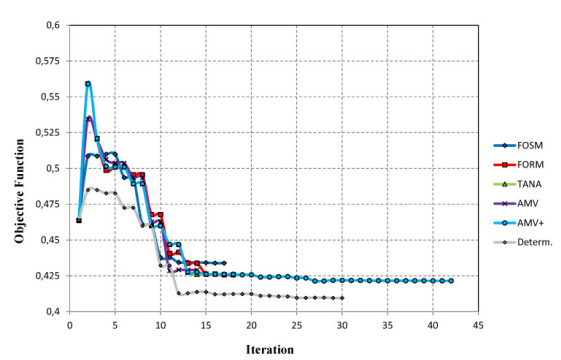

Figure 9: 25 bar 2D objective function.

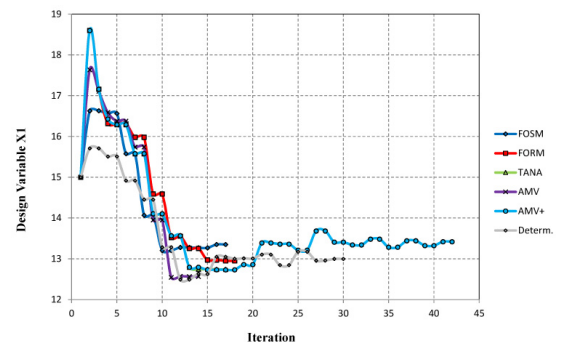

Figure 11: Convergence of design variable $x_{1}$.

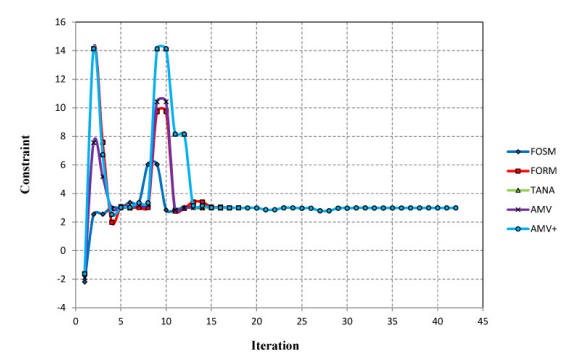

Figure 10: 25 bar 2D reliability constraint.

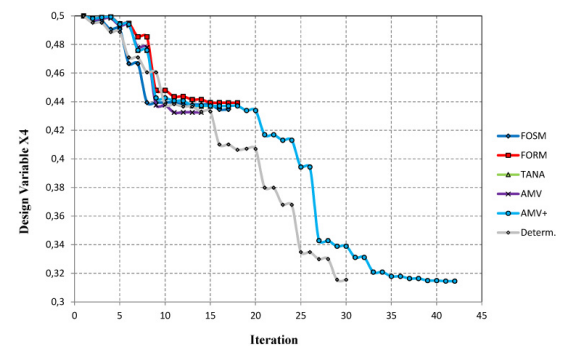

Figure 12: Convergence of design variable $x_{4}$.

As is shown in table 3 and figures 9 to 12 , the five reliability methods reach similar design variable and objective function values, with a slightly higher objective function value in the case of the FOSM method, with a relative increase of $2 \%$. The reason of this phenomenon is the different reliability index obtained with the FOSM method with respect to the others. It is also remarkable the fact that the deterministic optimization reaches a value only $4 \%$ lower. This is due to the low sensitivity that the loads have with respect to the structural displacements. In the AMV+ case, as it is shown in figure 9, the number of needed iterations is twice as much as the other methods, but in the last 30 the algorithm is converging around the neighbourhood of the solution, so the convergence is similar in all the cases, reaching acceptable values over the $15^{\text {th }}$ iteration. Focusing now on the number of needed evaluations, the FOSM and AMV methods are the least expensive, with only over 1100. TANA follows them with over 58,500 and FORM and AMV+ are the more expensive, with 182,000 and 213,000 , respectively.

\subsection{Twenty-five bar 3D truss}

Figure 13 shows the geometry of a 25 bar 3D truss structure. Four loads are applied in nodes $1,2,3$ and 6 with components $(0.1 P, P,-P),(0, P,-P),(0.05 P, 0,0)$ 


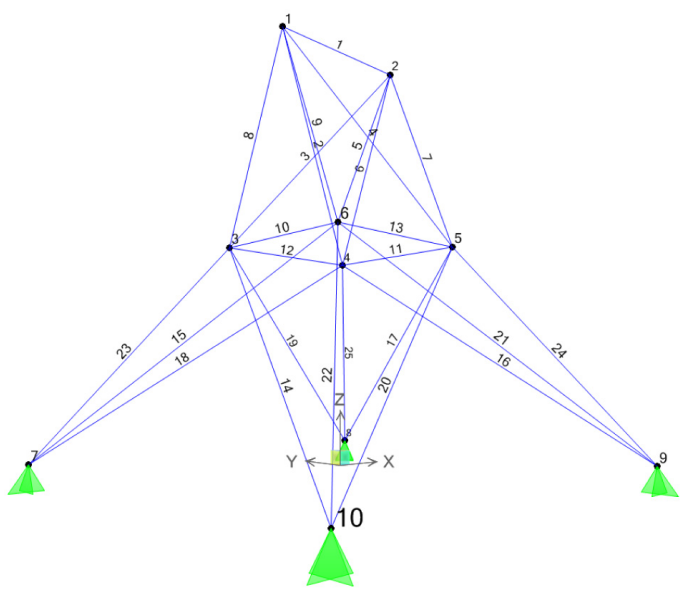

Figure 13: Twenty-five bar 3D truss structure.

and $(0.06 P, 0,0)$, respectively. The value $\mathrm{P}$ is taken as a random variable with a Gumbel distribution where the mean value is $25.58 \mathrm{kN}$ and the COV is 0.15 . The material is aluminum and its properties are the same as in the section 4.1. Design variables have an initial value of $19.634 \mathrm{~cm}^{2}$, and are constrained by a lower and upper bound, with values $0.636 \mathrm{~cm}^{2}$ and $50 \mathrm{~cm}^{2}$, respectively. The maximum horizontal displacement allowed on node 2 in the $y$ axis is $-0.889 \mathrm{~cm}$.

In this case, different results are obtained depending on the method (figures 14 to 17 and table 4). TANA and AMV+ methods reach similar objective function values, 0.213 . In the case of the FOSM method the result obtained has an increase

Table 4: Twenty-five bar 3D truss results summary.

\begin{tabular}{lccccc}
\hline Method & FOSM & TANA & AMV & AMV+ & Determ. \\
\hline$x_{1}$ & 0.636 & 0.636 & 9.638 & 0.636 & 0.636 \\
$x_{2}$ & 6.406 & 0.636 & 8.173 & 0.636 & 0.636 \\
$x_{3}$ & 21.895 & 23.312 & 40.417 & 23.905 & 12.332 \\
$x_{4}$ & 0.636 & 1.451 & 0.636 & 0.636 & 0.636 \\
$x_{5}$ & 8.132 & 12.909 & 23.551 & 11.583 & 6.780 \\
$x_{6}$ & 6.978 & 4.953 & 11.219 & 5.117 & 2.709 \\
$x_{7}$ & 6.621 & 1.182 & 0.839 & 1.054 & 0.636 \\
$x_{8}$ & 21.895 & 25.224 & 50 & 25.094 & 13.479 \\
$F$ & 0.250 & 0.214 & 0.431 & 0.213 & 0.115 \\
Iterations & 41 & 47 & 27 & 45 & 58 \\
Evaluations & 3051 & 160,857 & 2668 & 153,267 & 1386 \\
\hline
\end{tabular}




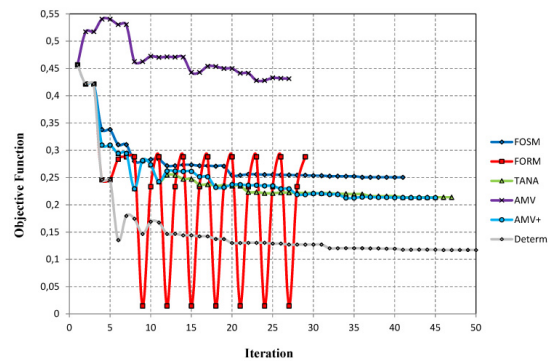

Figure 14: 25 bar 3D objective function.

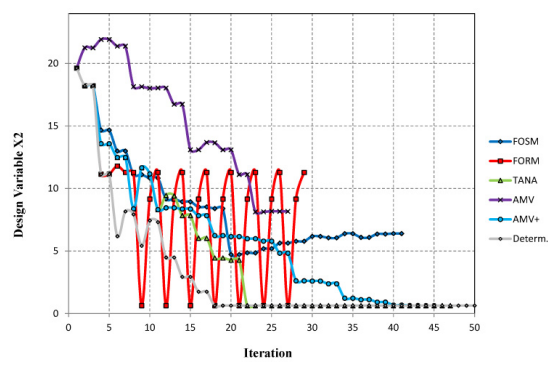

Figure 16: Convergence of design variable $x_{2}$.

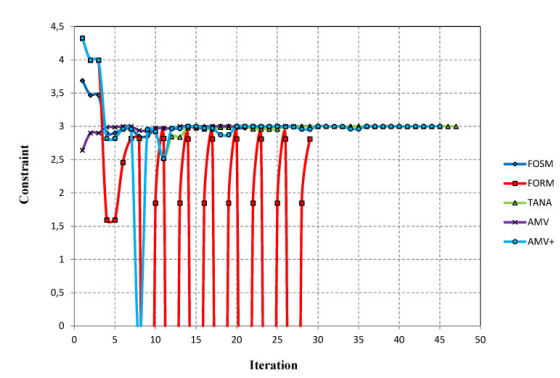

Figure 15: 25 bar 3D reliability constraint.

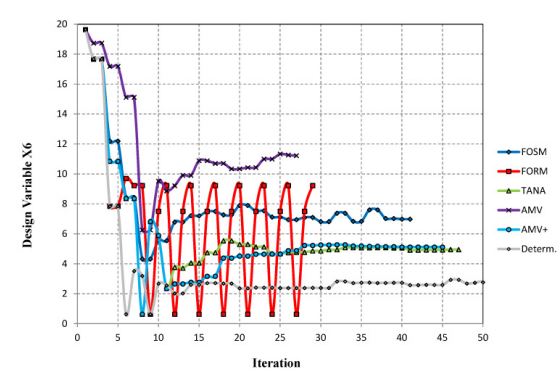

Figure 17: Convergence of design variable $x_{6}$.

of $17 \%$, but in the AMV case, the objective function doubles those values, reaching 0.431 . The reason is the different reliability index obtained with the AMV and FOSM method with respect to the others; for the initial point, the reliability index of each one of those methods has a value of 2.64 and 3.68, respectively, instead of 4.32 obtained with FORM, TANA and AMV+. This means that the probability of failure is higher and, therefore, the design variables and, consequently, the objective function will increase. It is also remarkable the fact that the FORM method does not reach convergence. As is shown in figure 14, from $8^{\text {th }}$ iteration starts to oscillate into three cyclical iterations. Deterministic optimization converges into a result $50 \%$ less than the obtained by RBDO.

All the methods reach convergence with a similar number of iterations with around 45, except AMV that finishes with only 27 iterations. Deterministic needs 58. Also, the most expensive methods, in terms of number of evaluations, are TANA and AMV+ with 160,000 and 153,000, respectively. The less computational intensive methods are the FOSM and AMV, with 3000 and 2600 evaluations, respectively. 


\section{Conclusions}

In this work, a review of some existing procedures of reliability analysis and their capabilities has been carried out in a RBDO framework. Three examples have been used to illustrate the performance of the methods employed in the study. Finally, some conclusions can be drawn.

As a general conclusion it can be said that depending on the reliability method used, the results of the RBDO can have slight changes. In particular, FOSM and AMV methods converge to less accurate values of reliability index than the other methods. This fact has an influence in the optimization results, which offer different values for the objective function.

Also, some differences were appreciated in terms of computational cost. FOSM and AMV are the fastest methods and FORM is the one requiring most iterations and does not always converge. Finally, AMV+ and TANA offer a good compromise between accuracy and efficiency.

\section{Acknowledgements}

The research leading to these results has received funding from the Spanish Ministry of Science and Innovation (Ministerio de Ciencia e Innovación) under grant agreement DPI2010-16238. The authors fully acknowledge the support received.

\section{References}

[1] Cornell, C.A., A probability-based structural code. Journal of the American Concrete Institute, 66(12), pp. 974-985, 1969.

[2] Hasofer, A. and Lind, N., Exact and invariant second moment code format. $J$ Eng Mech Div-ASCE, 100(1), pp. 111-121, 1974.

[3] Xu, S. and Grandhi, R., Effective two-point function approximation for design optimization. AIAA journal, 36, pp. 2269 - 2275, 1998.

[4] Yao, W., Chen, X., Luo, W., Van Tooren, M. and Guo, J., Review of uncertainty-based multidisciplinary design optimization methods for aerospace vehicles. Progress in Aerospace Sciences, 47(6), pp. 450-479, 2011.

[5] Yang, R., Chuang, C., Gu, L. and Li, G., Numerical experiments of reliabilitybased optimization methods. 45 th AIAA/ASME/ASCE/AHS/ASC Structures, Structural Dynamics and Materials Conference, 2004.

[6] Aoues, Y. and Chateauneuf, A., Benchmark study of numerical methods for reliability-based design optimization. Structural and Multidisciplinary Optimization, 41(2), pp. 277-294, 2009.

[7] Youn, B.D. and Choi, K.K., Selecting probabilistic approaches for reliabilitybased design optimization. AIAA journal, 42(1), pp. 124-131, 2004.

[8] Burton, S. and Hajela, P., Efficient reliability-based structural optimization through most probable failure point approximation. 45th AIAA/ASME/ASCE/AHS/ASC Structures, Structural Dynamics and Materials Conference, 2004. 